DRAFT VERSION JULY 7, 2021

Preprint typeset using LATEX style emulateapj v. 5/2/11

\title{
RICH KOZAI-LIDOV DYNAMICS IN AN INITIALLY THIN AND ECCENTRIC STELLAR DISC AROUND A SUPERMASSIVE BLACK HOLE
}

\author{
JAROSLAV HAAS AND LADISLAV ŠUBR \\ Charles University in Prague, Faculty of Mathematics and Physics, Astronomical Institute, V Holešovičkách 2, Praha, CZ-18000, Czech Republic \\ Draft version July 7, 2021
}

\begin{abstract}
There is growing evidence of star formation in the vicinity of supermassive black holes (SMBH) in galactic nuclei. A viable scenario for this process assumes infall of a massive gas cloud towards the SMBH and subsequent formation of a dense accretion disc which gives birth to the young stars. Numerical hydrodynamical models indicate that this star formation process is rather fast and it precedes full circularization of the accretion flow, i.e. the new stars are born on elliptic orbits. By means of direct numerical $N$-body modeling, we show in this paper that the non-zero eccentricity of the stellar discs around the SMBH leads to an onset of various types of the Kozai-Lidov oscillations of a non-negligible subset of individual orbits in the disc, showing a remarkable robustness of this classical mechanism. Among others, we demonstrate that under certain circumstances, presence of an additional spherical cluster (which is generally known to damp Kozai-Lidov oscillations) may trigger such oscillations due to affecting the internal flow of the angular momentum through the disc. We conclude that the Kozai-Lidov oscillations are capable to substantially modify the initial structure of the disc (its thickness and distribution of eccentricities, in particular).
\end{abstract}

Subject headings: Galaxy: nucleus — stars: kinematics and dynamics — celestial mechanics

\section{INTRODUCTION}

The discovery of a young stellar disc in the central parsec of the Milky Way (Levin \& Beloborodov 2003) and observations of a similar structure in our neighboring galaxy M31 in Andromeda (Bender et al. 2005) suggested the possibility that flattened stellar structures might represent a generic component of galactic nuclei. Since then, many aspects of the dynamical evolution of such systems have been investigated mostly in the context of the disc in the center of the Milky Way which is by far the best observationally accessible because of its proximity.

Diffusive processes due to two-body relaxation in an isolated disc of stars orbiting a supermassive black hole (SMBH) were analyzed, e.g., in Alexander et al. (2007) or Cuadra et al. (2008). These works described gradual thickening of the disc and growth of eccentricity of the individual stellar orbits. Two-body relaxation among the stars of the disc was also the key elementary process beyond the evolution of the radial structure of the disc studied by Subr \& Haas (2014). Resonant relaxation among the stellar orbits forming the disc was discussed by Tremaine (1998). Later on, this process between the disc and an embedding spherical star cluster was addressed by Kocsis \& Tremaine (2011) who found that this process may lead to a significant warp of the disc. Angular momentum transfer through an eccentric stellar disc embedded in a spherical cluster was investigated by Madigan et al. (2009). Other works (e.g. Nayakshin et al. 2006; Subr et al. 2009; Gualandris et al. 2012) considered various additional external sources of a perturbative gravitational potential in order to explain the properties of the observed structures of young stars in the Galactic Center through secular dynamics.

Some of the above introduced works consider the stellar disc to be formed by initially eccentric orbits. According

E-mail: haas@sirrah.troja.mff.cuni.cz; subr@sirrah.troja.mff.cuni.cz

${ }^{1}$ On the scale of planetary systems, this topic was studied even earlier (see Stewart \& Ida 2000, and references therein). to works of Bonnell \& Rice (2008), Wardle \& Yusef-Zadeh (2008), Hobbs \& Nayakshin (2009) or Mapelli et al. (2012), such a setting is a probable outcome of the currently most widely accepted formation scenario for the young stellar disc in the center of the Milky Way via partial capture of an infalling massive gaseous cloud by the central SMBH. Models of eccentric disc of young stars were also considered to explain the observed properties of the nucleus of M31 (e.g. Tremaine 1995; Peiris \& Tremaine 2003). Presence of initially eccentric orbits in the disc broadens the variety of possible modes of angular momentum exchange throughout the disc. In our paper, we focus on the numerous effects of the Kozai-Lidov dynamics in the potential of the disc itself and of the embedding spherical potential.

\section{KOZAI-LIDOV DYNAMICS}

In contrast to the classical, purely self-gravitating star clusters, the motions of stars in central mass dominated systems are highly regular. This leads to their mutual interaction on time-scales greatly exceeding the typical orbital periods around the center. In order to describe the resulting slow (secular) evolution of their orbits, it is very useful to think of the whole system as averaged over one orbital revolution. This approach (commonly called the averaging technique) has been developed in the perturbation theory of the celestial mechanics and represents a well justified and widely used tool for such a problem which relies on the classical Hamiltonian formalism (see, e.g., Morbidelli 2002; Bertotti et al. 2003). In the sense of the averaging technique, the individual orbits can be also thought as wires exerting torques on one another which directly affect their angular momenta; note, however, that energy (semi-major axis) is conserved within the averaging approximation.

The averaging technique can be straightforwardly used to determine the secular evolution of a Keplerian orbit under the perturbative influence of a flattened potential. Such a potential can represent either some rather continuous matter distribution (e.g. gaseous structures) or, in the context of the 

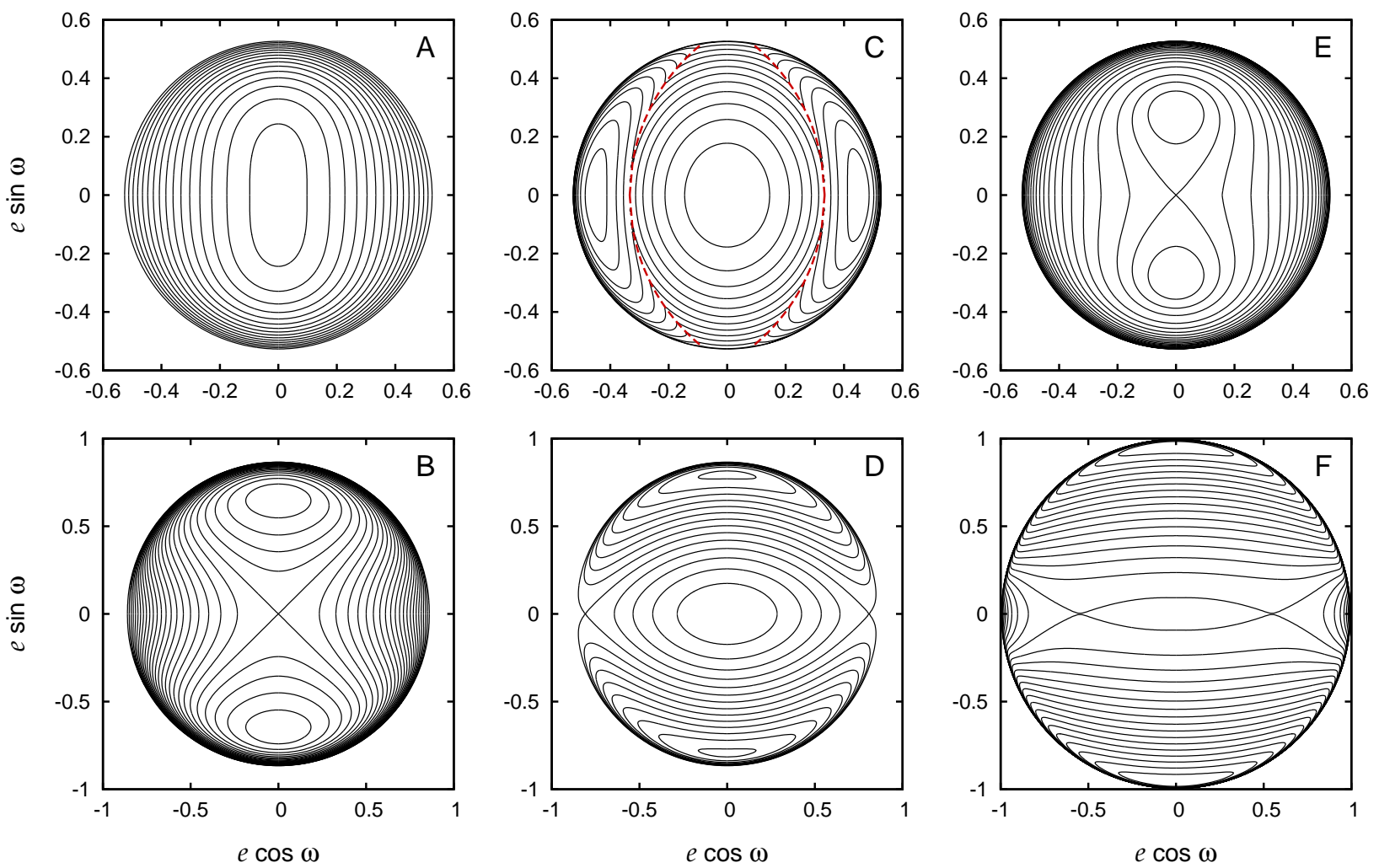

FIG. 1.- Isocontours of the averaged perturbative potentials in the $e-\omega$ space for several different settings. The perturbing potential is due to an infinitesimally thin ring of radius $R_{\mathrm{d}}$ in panels $\mathrm{A}$ through $\mathrm{C}$; in panel $\mathrm{D}$ the perturbing potential is a superposition of potentials of a ring and a spherical cluster with radial density profile $\varrho(r) \propto r^{-7 / 4}$ and mass $M_{\mathrm{C}}=1.5 M_{\mathrm{d}}$ within the radius $R_{\mathrm{d}}$. In panels $\mathrm{E}$ and $\mathrm{F}$ the source of the perturbing potential is a razor-thin disc of constant surface density and outer radius $R_{\mathrm{d}}$. Specific values of orbit semi-major axis, $a$, and the Kozai integral, $c$, are: A: $a=0.48 R_{\mathrm{d}}$, $c=0.85$; $\mathrm{B}$ : $a=0.48 R_{\mathrm{d}}, c=0.5 ; \mathrm{C}: a=1.50 R_{\mathrm{d}}, c=0.85 ; \mathrm{D}: a=0.48 R_{\mathrm{d}}, c=0.5 ; \mathrm{E}: a=0.48 R_{\mathrm{d}}, c=0.85 ; \mathrm{F}: a=0.48 R_{\mathrm{d}}, c=0.1$. Dashed line in panel $\mathrm{C}$ corresponds to orbits which intersect the perturbing ring. Note different ranges of the boxes in the upper and lower panels which reflect different maxima of eccentricity for different values of $c$.

averaging technique, the averaged potential of a body or a disc of bodies. The common feature of the flattened disturbing potentials is that they decrease the degree of symmetry of the unperturbed Keplerian potential. As a result, the vector of angular momentum of the Keplerian orbit is no longer an integral of motion, permitting various ways of its evolution, either periodic or chaotic.

A systematic study of the secular evolution of a Keplerian orbit due to presence of a perturbing flattened potential began with the works of Kozai (1962) and Lidov (1962) who studied the simplest form of this problem, the secular evolution of the hierarchical three-body problem, i.e. a system in which two bodies form a well-defined binary and the third body orbits around this binary staying well separated from it. In these pioneering works, one of the components of the inner binary has been considered massless and the orbit of the distant third body circular, making its (averaged) perturbing potential axially symmetric and keeping the projection of the angular momentum of the test orbit onto the symmetry axis an integral of motion. Due to conservation of the orbital semi-major axis, $a$, of the test orbit (within the averaged approximation), the conserved projection of the angular momentum may be replaced by the so-called Kozai integral, $c \equiv \sqrt{1-e^{2}} \cos i$, where $e$ and $i$ are eccentricity and inclination of the test orbit measured from the symmetry axis, respectively. Hence, the Kozai integral enables us to eliminate $i$ (say) from further considerations. Since the nodal longitude, $\Omega$, of the orbit does not affect its evolution due to symmetry of the potential, the remaining problem is to solve coupled equations for secular evolution of eccentricity and argument of pericenter, $\omega$.

Within the averaging approximation, the perturbing potential averaged over one Keplerian revolution around the center is another (third) integral of motion. When the concern is about the trajectory in the phase space rather than about temporal evolution of the orbital elements, it is possible to bypass the problem of solving differential equations by directly investigating isocontours of the averaged perturbing potential in the $e-\omega$ space which represent the allowed trajectories. The exemplary diagrams that reveal qualitatively different configurations are shown in Figure 1

We start with the classical setup, when the perturbation is due to a distant third body on a circular orbit of radius $R_{\mathrm{d}}$ and the multipole expansion of its averaged potential in ratio $a / R_{\mathrm{d}}$ is truncated after the quadrupole term (for details, see, e.g., Kozai 1962). Panel A corresponds to initial conditions for which the test orbit does not undergo any significant oscillations of eccentricity and $\omega$ rotates in the full interval $\langle 0,2 \pi\rangle$, following the simple oval-shaped isocontours. Such a case occurs whenever the orbit fulfills the criterion $c>\sqrt{3 / 5}$ (the so-called Kozai limit which corresponds to $i \lesssim 39.2^{\circ}$ for the circular orbit). The topology of the isocontours changes dramatically for $c \leq \sqrt{3 / 5}$ (panel B). Two new stationary points at $\omega=\pi / 2$ and $3 \pi / 2$ appear and a separatrix curve emerging from the origin separates two regions in which $\omega$ librates in a limited interval from the outer region in which it still circulates. The two stationary points correspond to solu- 
tions during which two angles, $\varpi \equiv \Omega+\omega$ and $\Omega$ are in $1: 1$ resonance, leaving $\omega$ constant during the evolution. For this reason, this type of topology is commonly referred to as resonant and we will adopt this nomenclature further on. The most important feature of the resonant topology are high-amplitude oscillations of eccentricity, forcing the orbit to a very high eccentricity state no matter how small its initial eccentricity was. In the honor of their discoverers, these oscillations are commonly called Kozai-Lidov oscillations and the whole phenomenon Kozai-Lidov resonance (cycles; mechanism). Due to existence of the Kozai integral $c$, the test orbit is thus oscillating between two extremes: (i) low eccentric and highly inclined with respect to the plane of symmetry of the potential (perturbing orbit), and (ii) highly eccentric and nearly coplanar. Another immediate consequence of the Kozai integral is that the inclination of the test orbit may never cross value $i=\pi / 2$, in other words, the orbital motion of the test particle around the central mass remains either prograde or retrograde with respect to the motion of the third body, never changing this sense.

Over the many decades since the original discovery, many other variants and generalizations of the classical KozaiLidov problem have been studied extensively. Among them, let us briefly comment on those which we found to be relevant for the numerical models presented below. It was shown by Bailey et al. (1992) and further systematically studied by Thomas \& Morbidelli (1996) or Gallardo et al. (2012), that the dynamics in the case when the perturbing body is inside the orbit of the test particle is very much different in comparison with the classical setting (panel C in Figure 1). Most notably, the initially circular orbit is not an unstable solution of the problem, i.e., in order to undergo significant oscillations, the test orbit must possess (or reach due to some other processes) a certain non-zero eccentricity. Furthermore, in contrast to the classical setting, the stationary points in the resonant topology are located at $\omega=0$ and $\pi$ and, for lower values of the Kozai-Lidov integral, even more libration regions may appear. Let us also note that if the test orbit undergoes significant oscillations of its eccentricity, it may cross the orbit of the perturbing body at which point the assumptions of the approximation are no longer valid. Therefore, the affected isocontours of the perturbing potential may not correctly represent the real dynamics of the test orbit. The dashed line in panel $\mathrm{C}$ connects the points for which this occurs.

It was further shown that the Kozai-Lidov oscillations can be inhibited by embedding the three-body system into some (sufficiently strong) spherical gravitational potential (e.g., Ivanov et al. 2005; Subr et al. 2007; Haas et al. 2011). The reason for this is the precession of the argument of pericenter $\omega$ of the test orbit caused by the spherical potential which disturbs the Kozai-Lidov resonance. In terms of isocontours of the perturbing potential, superposition of the potential of the ring with the spherical component leads to formation of an inner rotational region which we illustrate in panel D in Figure 1. We can see that, similarly to the case of the interior perturber, it is necessary for the test orbit to possess some nonzero eccentricity in order to undergo significant Kozai-Lidov oscillations. Moreover, increasing strength of the spherical perturbing potential leads to a decrease of the limiting value of the Kozai-Lidov integral, $c$, below which the resonance occurs, i.e. it decreases the volume of the resonant part of the phase space.

In our numerical models, the source of the perturbing po- tential is represented by a moreorless thin disc rather than an infinitesimally thin ring. As an example, we plot isocontours for a razor-thin axially symmetric disc of a constant surface density ranging from $r=0$ to $r=R_{\mathrm{d}}$ in panels $\mathrm{E}$ and F of Figure 1 (see, e.g., Vokrouhlický \& Karas (1998) or Subr \& Karas (2005) for a general discussion of this topic). Panel E demonstrates that such a disc is a stronger perturbation than a ring (averaged body) in the sense that for identical values of parameters $\left(R_{\mathrm{d}}, a\right.$ and $\left.c\right)$, the topology is resonant for the disc while it is still non-resonant in the case of the ring (panel A in Figure 1). Another qualitatively new feature of the isocontours for the case of a disc-like perturbation which appears for sufficiently low values of $c$ is the existence of the inner rotational region (panel F).

When the axial symmetry of the perturbing potential is lost by considering the perturbing body to revolve around the central mass on an eccentric orbit or by considering an eccentric stellar disc, the Kozai 'integral' $c$ is no longer an integral of motion. Hence, dimension of the manifold covered densely by the trajectory in the phase space is higher in comparison to the classical setup and evolution of the test orbit may be very complex (see, e.g., Katz et al.|2011; Lithwick \& Naoz 2011; Naoz et al. 2013; Li et al. 2014a.b). One of the qualitatively new phenomena are orbital flips, i.e. changes of the sense of the orbital motion along the test orbit from prograde to retrograde and vice versa. During these flips, eccentricity of the test orbit can reach as extremely high values as $e \sim 1-10^{-6}$ (Li et al. 2014a). Due to non-existence of the Kozai-Lidov integral, it is also not possible to use the isocontours plotted solely in the $e-\omega$ space of the perturbing potential as a guide through the evolution of the test orbit in contrary to the case of axially symmetric perturbations. On the other hand, when the rate of change of $c$ is sufficiently slow, the orbit actually follows the isocontours of the perturbing potential for a given value of $c$, i.e. it slowly migrates from one topology to another. As the evolution of $c$ is determined by the octupole term (and eventually higher order terms) of expansion of the averaged perturbing potential, we refer to this process as the octupole modulation of the classical Kozai-Lidov cycles (or simply octupole Kozai-Lidov cycles) further on.

\section{NUMERICAL RESULTS}

Having briefly summarized the key aspects of the KozaiLidov dynamics, let us now turn to identification of its footprints in our numerical models.

\subsection{Model and method}

First, we introduce several numerical models of a stellar disc around a SMBH which we used to study the Kozai-Lidov dynamics. The central SMBH is simulated by a fixed Keplerian potential of point mass $M_{\bullet}$ which we also use as a definition of mass unit, i.e. $M_{\bullet}=1 M_{\mathrm{u}}$. The stellar disc of total mass $M_{\mathrm{d}}$ is represented by 500 particles of equal mass. Except for one model, we considered $M_{\mathrm{d}}=0.00125 M_{\bullet}$ (see Table 1). Semi-major axes are generated according to the distribution function $n_{a}(a) \propto a^{-1}$ in $\left\langle 10 r_{\mathrm{u}}, 100 r_{\mathrm{u}}\right\rangle$ which approximately corresponds to a disc surface density $\propto r^{-2}$. Here, $r_{\mathrm{u}}$ represents an arbitrary length unit and, together with $M_{\mathrm{u}}$ it can be used for a natural definition of the unit of time ${ }^{2}$ , $t_{\mathrm{u}} \equiv \sqrt{r_{\mathrm{u}}^{3} / G M_{\mathrm{u}}}$, where $G$ stands for the gravitational con-

${ }^{2}$ For a natural target system, the Galactic Center, our models scale as: $M_{\mathrm{u}} \approx 4 \times 10^{6} M_{\odot}, r_{\mathrm{u}} \approx 0.004 \mathrm{pc}$ and $t_{\mathrm{u}} \approx 1.9 \mathrm{yr}$. 

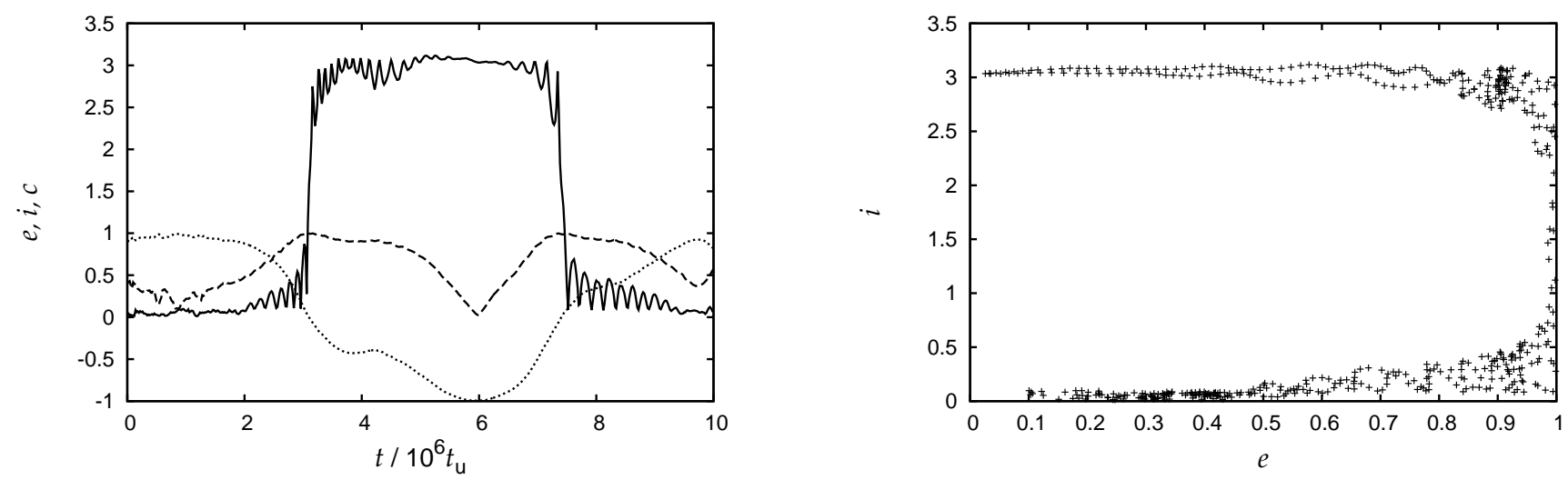

FIG. 2.- A flipping orbit from model M3. Left panel: Evolution of eccentricity (dashed line), inclination (solid) and the Kozai-Lidov integral (dotted). Right panel: Evolutionary track of that orbit in the $e-i$ space (cf. right panels of Figures 3 and 4 in Li et al. 2014a).

TABLE 1

VARIABLE PARAMETERS OF THE MODELS

\begin{tabular}{c|cccc} 
identification & $e_{0}$ & $M_{\mathrm{d}} / M_{\bullet}$ & $M_{\mathrm{c}} / M_{\mathrm{d}}$ & orientation \\
\hline M1 & 0 & 0.00125 & 0 & - \\
M2 & 0.4 & 0.00125 & 0 & random \\
M3 & 0.4 & 0.00125 & 0 & aligned \\
M4 & 0.4 & 0.00125 & 10 & aligned \\
M5 & 0.4 & 0.00125 & 100 & aligned \\
M6 & $\langle 0,0.9\rangle$ & 0.00125 & 0 & aligned \\
M7 & $\langle 0,0.9\rangle$ & 0.00125 & 10 & aligned \\
M8 & $\langle 0,0.9\rangle$ & 0.00612 & 0 & aligned \\
M9 & $\langle 0,0.9\rangle$ & 0.00612 & 4 & aligned
\end{tabular}

Description: $e_{0}$ is the initial eccentricity of the orbits; in the case of models M6 - M9, eccentricity is a function of the semi-major axis according to formula $e_{0}=0.9\left(a-a_{\min }\right) /\left(a_{\max }-a_{\min }\right) ; M_{\bullet}, M_{\mathrm{d}}$ and $M_{\mathrm{c}}$ are mass of the central potential, disc and spherical cluster, respectively; 'aligned' orientation means that all orbits have initially common directions of the apsidal lines, while 'random' corresponds to the case of uniformly distributed longitude of the ascending node and argument of pericenter.

stant. Initial inclinations are generated according to the distribution function $\propto \sin i$ in an interval $\left\langle 0,2^{\circ}\right\rangle$, i.e. the normal vectors of the orbital planes are uniformly distributed within a cone with the half-opening angle $2^{\circ}$. Values of the initial eccentricities and orientations of the orbits are described in Table 1 Finally, an optional component of the model is a spherically symmetric gravitational potential $\Phi_{\mathrm{c}} \propto \sqrt{r}$ which corresponds to a smooth distribution of mass with density $\varrho_{\mathrm{c}}(r) \propto r^{-3 / 2}$. It stands for a spherically symmetric star cluster parametrized by its mass, $M_{\mathrm{c}}$, enclosed within the radius $100 r_{\mathrm{u}}$, i.e. approximately within the radial domain of the disc.

We concentrated solely on the dynamics of the studied system, i.e. all stars were treated as point masses. Equations of motion were integrated by means of the $N$-body integration code NBODY6 (Aarseth 2003) which has been modified by addition of the external potential of the SMBH and the spherical cluster. We have also added an option for monitoring the minimal value of the radial coordinate (i.e. distance to the $\mathrm{SMBH})$ for each particle.

\subsection{Kozai-Lidov oscillations}

In our calculations, we have met various modes of the Kozai-Lidov dynamics, depending on the particular setups of the integrated models. In the following paragraphs, we demonstrate them on selected individual trajectories. Let us, however, mention that, due to the stochastic nature of the considered $N$-body system, the sample orbits lack the purity of secular evolution often seen within the systems investigated in the scope of the celestial mechanics. Still, the orbits presented below belong to those which enable us to identify the particular mode of the Kozai-Lidov dynamics. Beside them, we have observed many other orbits undergoing some kind of coupled eccentricity and inclination oscillations which, however, were difficult to be undoubtedly categorized.

The most prominent effect which is invoked by a non-zero eccentricity of the stellar disc is the flipping of the orbits in its innermost parts. This kind of evolution can be best observed in model M3 which lacks the additional spherical potential; an example of a flipping orbit is shown in Figure 2 A rather periodic secular evolution of $c$ (left panel, dotted line) indicates that the octupole term of the perturbing potential plays an important role. More specifically, both the slow secular change of eccentricity (dashed line) as well as the nearly step-wise fashion change of inclination (solid line) are characteristic for the so-called coplanar flipping phenomenon (Li et al. 2014a). An additional piece of evidence for this particular type of the octupole modulation of the classical Kozai-Lidov cycles is the shape of the area covered by the evolutionary track of the flipping orbit in the $e-i$ space (right panel; cf. Li et al. 2014a, Figure 4).

Once the disc is embedded in a spherical cluster, its evolution changes. The coplanar flipping in the innermost parts of the disc becomes less frequent, as fewer orbits can reach the octupole Kozai-Lidov resonances. Weakening of the systematic secular evolution leads to promotion of chaotic pattern of orbital trajectories in the phase space. An example of the better-behaved (i.e. less chaotic) orbits is displayed in Figure 3. We can see that it first evolves rather stochastically in the complex $N$-body setting, however, showing a systematic decrease of the semi-major axis $a$ (left panel). At $t \approx 2 \times 10^{6} t_{\mathrm{u}}$, the fashion of the evolution changes. Semimajor axis $a$ stops decreasing and remains roughly constant further on. On the other hand, the value of the Kozai-Lidov integral $c$ (right panel, dotted line) starts to decrease in a very smooth way from nearly unity to a near-zero value reached at $t \approx 3 \times 10^{6} t_{\mathrm{u}}$. As the time-scale of this decrease covers $\sim 10^{4}$ orbital periods, it appears that it is of a secular rather than stochastic nature, presumably being a fragment of an octupole Kozai-Lidov cycle in the overall non-axisymmetric potential of the disc. When the Kozai-Lidov integral $c$ stops decreasing, it remains roughly constant for the rest of the in- 

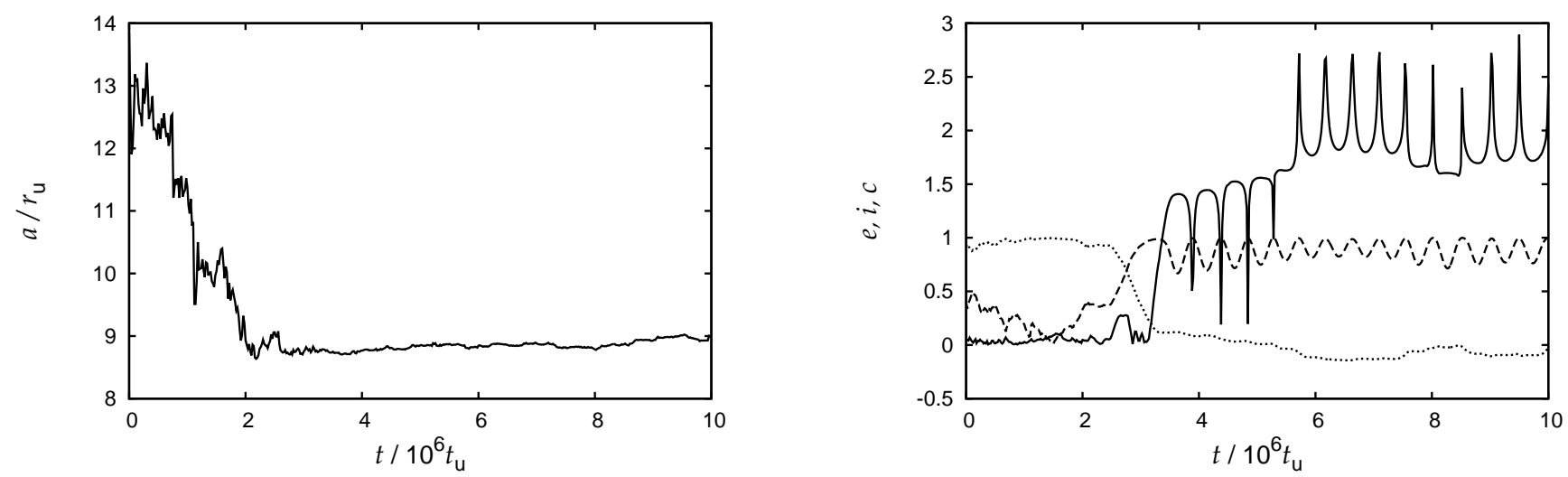

FIG. 3.- An oscillating orbit in model M4. Left: Evolution of the semi-major axis. Right: Evolution of the eccentricity (dashed line), inclination (solid) and the Kozai-Lidov integral (dotted).
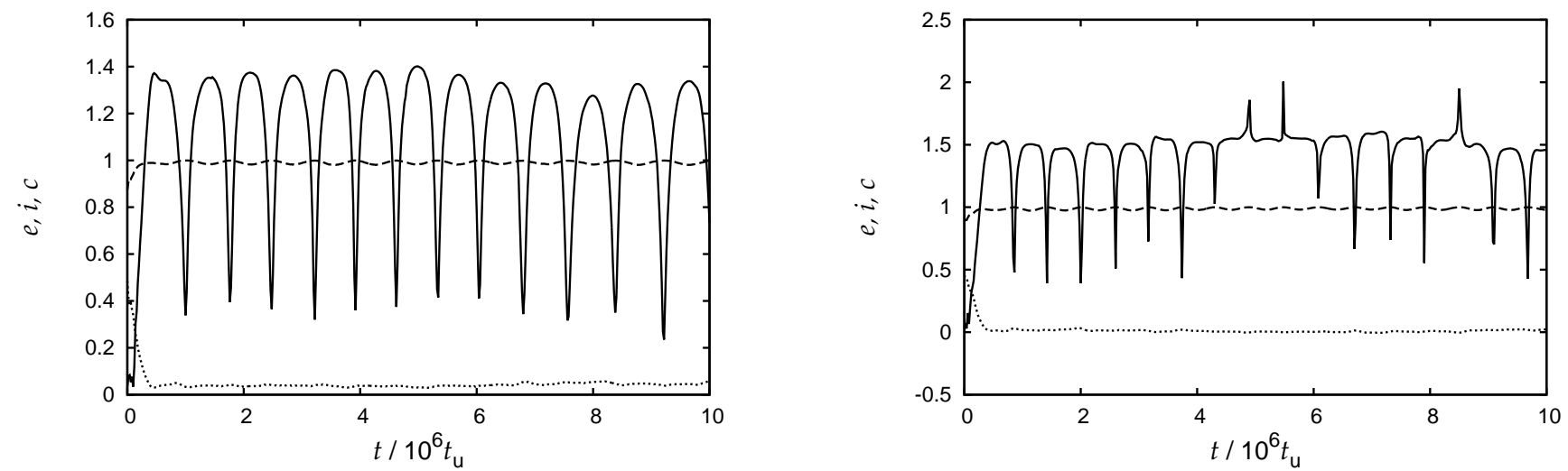

FIG. 4.- Two orbits from model M9 undergoing the outer Kozai-Lidov cycles. Evolution of eccentricity, $e$, inclination, $i$ and the Kozai-Lidov integral, $c$, is plotted with dashed, solid and dotted line, respectively (cf. Figure 2 in Bailev et al. 1992).

vestigated time interval, showing only random-walk-fashion fluctuations. At the same time, mutually coupled periodic oscillations of eccentricity $e$ and inclination $i$ occur (right panel, dashed and solid line), during which $e$ reaches nearly unity in its maxima. This indicates that, from $t \approx 3 \times 10^{6} t_{\mathrm{u}}$ on, the orbit undergoes the classical Kozai-Lidov cycles in the global potential of the disc. We can further speculate that their onset occurred when the (initially very high) value of $c$ became sufficiently low for the Kozai-Lidov resonance to appear, i.e. for the topology of the perturbing potential isocontours to change from non-resonant to the resonant one (see Section 2). The orbital flip that took place at $t \approx 5.5 \times 10^{6} t_{\mathrm{u}}$ is likely to be a result of a random two-body encounter that changed the sign of the near-zero $c$. Note that oscillations of individual orbits were also reported by Madigan et al. (2009, Fig. 1) in a setup which is similar to our model M4. We suppose that also in their case, the driving mechanism was the Kozai-Lidov resonance.

Yet another type of oscillations occur in the outer parts of the disc when it is embedded in the spherical cluster (i.e. they were not present in an isolated disc). The affected orbits undergo the so-called outer Kozai-Lidov oscillations (e.g. Bailey et al. 1992; Thomas \& Morbidelli 1996; Gallardo et al. 2012). Examples of such oscillating orbits, which are particularly common in model M9, are shown in Figure 4 The presence of these orbits is, at a first glance, a rather unexpected result as the spherical potential of the clus- ter generally damps such resonant effects. However, in spite of the fact that the potential of the cluster indeed decreases volume of the resonant area in the phase space and pushes it to higher eccentricities, the same potential can modify the internal flow of angular momentum in the disc, increasing its ability to push the stellar orbits to high eccentricities, i.e. to the resonant area. The role of the spherical cluster in triggering the Kozai-Lidov cycles in the outer parts of the disc is visualized in Figure 5 which shows positions of stellar orbits in the $a-e$ space for models M8 (without the spherical cluster) and M9. In the former case, we see extreme eccentricities of several orbits from the inner parts of the disc which undergo the octupole Kozai-Lidov cycles (their pattern is similar to the example trajectory presented in Figure 2). However, when the spherical stellar cluster is included, the oscillating orbits at the inner edge of the disc are suppressed and we observe extreme eccentricities at the outer edge of the disc.

\subsection{Statistical view on the Kozai-Lidov oscillations}

A common feature of all of the above described oscillations are high values of eccentricity reached during its maxima. One of the methods how to estimate the effectivity of the individual models in producing high-eccentricity orbits is to evaluate the closest approaches of the stars to the central SMBH during the whole integration and construct their cumulative distribution function. The results for the models introduced in this paper are shown in Figure 6. In agreement with 

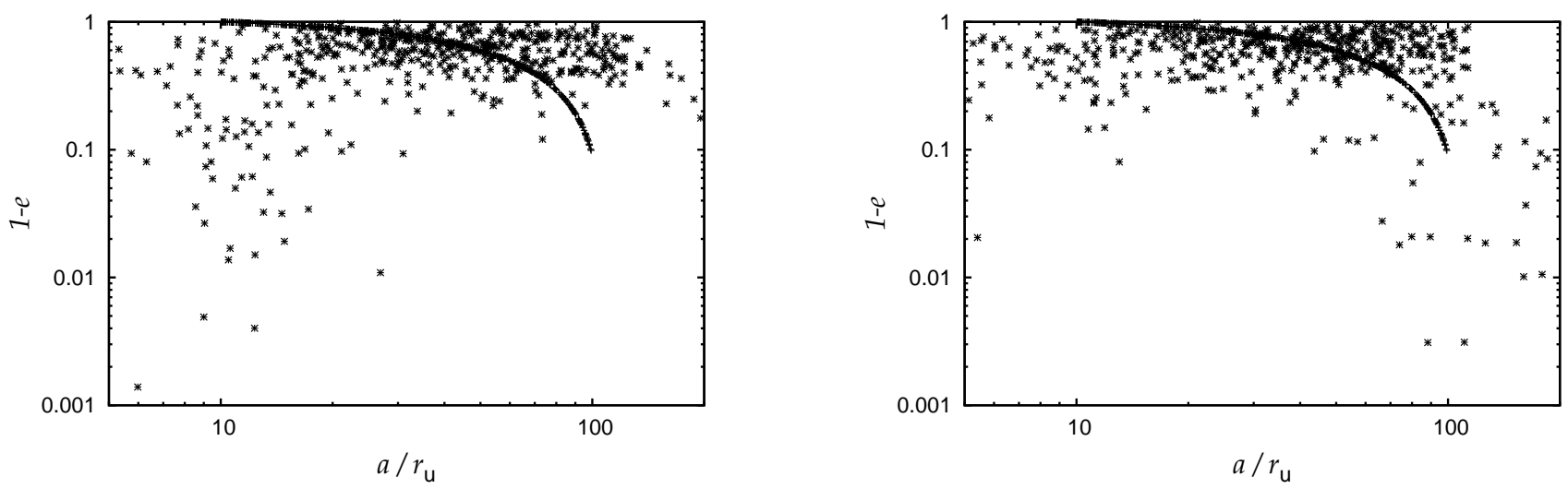

FIG. 5.- Distribution of stellar orbits in the $a-e$ space for models M8 (left) and M9 (right). Plus signs that form a continuous line represent the initial state, while the crosses show overplot of three states at $t=10^{5} t_{\mathrm{u}}, 10^{6} t_{\mathrm{u}}$ and $10^{7} t_{\mathrm{u}}$.

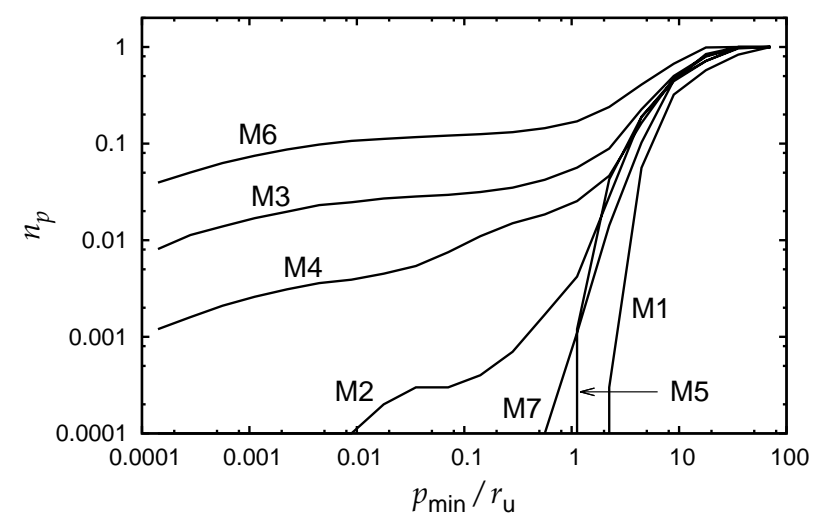

FIG. 6.- Cumulative distribution functions of the closest approaches, $p_{\min }$, of the stars to the SMBH in various models (averaged over 20 realizations in all cases).

intuition, there are no high-eccentricity orbits in the initially circular disc (M1). When the orbits are initially eccentric but randomly oriented (M2), some of them are pushed to high eccentricities (presumably) due to mutual interactions, however, their number is still very low. The situation changes when the orbits are initially aligned - in models M3 and M6, a large fraction of orbits reach eccentricities higher than 0.9. A substantial fraction of those in the inner parts of the disc undergo the coplanar flipping.

Subsequent models M4 and M5 share all but one parameter, $M_{\mathrm{c}}$, with model $\mathrm{M} 3$ and they demonstrate the damping effect of the spherical cluster. When $M_{\mathrm{c}}=10 M_{\mathrm{d}}$ (model M4), a non-negligible number of oscillating orbits still persist but, unlike for model M3, most of them are of the quadrupole nature (with $c$ conserved). Increasing further the mass of the spherical cluster (model M5) leads to a complete damping of Kozai-Lidov oscillations and the distribution of minima of pericenters becomes similar to models M1 and M2 with an axially symmetric disc. This result is in accord with Gualandris et al. (2012) who followed evolution of an eccentric disc embedded in a cluster of mass $M_{\mathrm{c}} \approx 140 M_{\mathrm{d}}$ and did not observe any periodic oscillations of orbital elements.

Finally, Figure 6also demonstrates sensitivity of the angular momentum transfer processes on the properties of the disc, in particular, the initial distribution of eccentricities. In the case of a standalone disc (i.e. not embedded in the spherical cluster), model M6 with eccentricities increasing from zero

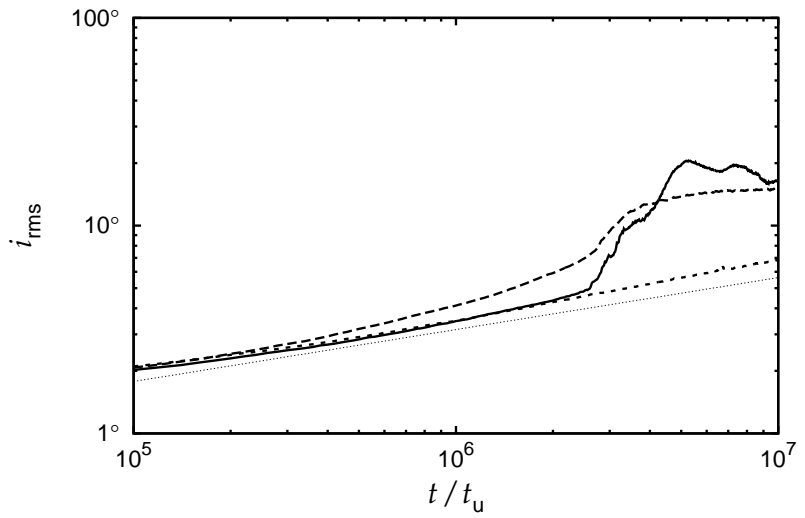

FIG. 7.- Evolution of the root-mean-square inclination in the disc for models M3, M4 and M2 (solid, dashed and dotted lines, respectively; averaged over 20 realizations). Thin dotted line represents slope $t^{1 / 4}$.

to 0.9 outwards produces by a factor of $\approx 5$ more extremely oscillating orbits than model M3 which is initially composed of equally eccentric orbits $\left(e_{0}=0.4\right)$. However, adding a spherical potential of mass $M_{\mathrm{c}}=10 M_{\mathrm{d}}$ leads to a complete damping of orbital oscillations in the case of disc with gradient distribution of eccentricities in contrary to the model with initially equal eccentricities, which is able to produce highly eccentric orbits in spite of the presence of the extended spherical potential (cf. models M7 and M4).

Let us mention that the production rate of high-eccentricity orbits in our models may be affected by the fact that we emulate the gravity of the spherical cluster by a smooth analytic potential. The orbit presented in Figure 3 however, indicates that the secular resonances may be able to trap some orbits even in the disturbing $N$-body environment. The particular displayed orbit undergoes systematic change of the KozaiLidov integral during the time interval $2.5 \times 10^{6} t_{\mathrm{u}} \lesssim t \lesssim$ $3 \times 10^{6} t_{\mathrm{u}}$. At the same time, it is mostly embedded in the disc, whose relaxation time is definitely shorter than the relaxation time of grainy spherical cluster of reasonable parameters. Furthermore, it was already suggested by Löckmann et al. (2009) that a grainy potential of the real astrophysical cluster is not as effective in damping the Kozai-Lidov resonance as the corresponding smooth potential. Hence, our estimates of the number of produced high-eccentricity orbits may serve as lower limits.

Another quantity which reflects the Kozai-Lidov oscilla- 
tions of individual orbits from the disc is a root-mean-square value of inclination, $i_{\text {rms }}$. In the case of an initially circular disc, it has been predicted theoretically and also verified by means of numerical models (e.g. Stewart \& Ida 2000) that $i_{\text {rms }}$ should grow in time approximately as $i_{\text {rms }} \propto t^{1 / 4}$ due to the two-body relaxation. As we show in Figure 7 (dotted line), also model M2 which represents an axially symmetric disc formed by randomly oriented eccentric orbits exhibits the same rate of evolution of $i_{\text {rms }}$. A qualitatively different evolution is observed in the case of model M3 (solid line) which is formed by aligned eccentric orbits - from $t \approx 3 \times 10^{6} t_{\mathrm{u}}$ on, we see an accelerated growth of $i_{\text {rms }}$. Let us note that such an accelerated growth of $i_{\text {rms }}$ has been reported already by Cuadra et al. (2008, Figure 3) who integrated a model similar to our one. Unlike these authors, who attributed this effect to resonant relaxation, we interpret this behavior as a manifestation of the Kozai-Lidov oscillations. In particular, in this case, when the disc is not embedded in the additional spherical potential, we distinguish majority of the oscillations to correspond to the octupole Kozai-Lidov cycles, often leading to the coplanar flipping during which inclinations of the orbits reach values very close to $\pi$. The accelerated growth of $i_{\text {rms }}$ is then a consequence of averaging over a sample of orbits which includes a certain number of those with large inclinations. Let us, however, point out that this growth of $i_{\text {rms }}$ does not necessarily lead to geometrical thickening of the disc. The orbits undergoing octupole Kozai-Lidov cycles reach extreme eccentricities at the stages of high $\sin i$, i.e. although being highly inclined, they are still embedded in the thin disc structure.

The picture, however, changes when the disc is embedded in the spherical cluster (model M4; dashed line in Figure 7 . In this case, the coplanar flips are substantially damped, which leads to a less prominent jump of $i_{\text {rms }}$ at $t \approx 3 \times 10^{6} t_{\mathrm{u}}$. On the other hand, evolution of $i_{\text {rms }}$ within model M4 deflects from the $t^{1 / 4}$ line already at $t<10^{6} t_{\mathrm{u}}$, which we found to be due to presence of the quadrupole Kozai-Lidov oscillations with conserved $c$. This, in contrary to the octupole modulated case, means that the oscillating orbits reach minima of their eccentricities at the high inclination stage, i.e. they spend non-negligible period of time above the equatorial plane and the value of $i_{\text {rms }}$ reasonably well represents the geometrical thickness of the disc.

\section{CONCLUSIONS}

We analyzed the orbital evolution of an initially thin and eccentric stellar disc around the central supermassive black hole $(\mathrm{SMBH})$. When the stellar orbits are initially randomly oriented, the overall evolution is similar to that of a circular disc. If the orbits share a common orientation of their apses, the evolution of the disc is significantly different, comprising a variety of the Kozai-Lidov oscillations of eccentricity and inclination of different types. These strongly depend on the particular initial setup; among all the possible variations, we have explicitly discussed the role of an embedding spherical cluster and the eccentricity distribution within the disc.

The onset of the Kozai-Lidov oscillations appears to be a rather generic process occurring in eccentric stellar discs. Its systematic nature can successfully compete with the chaotic two-body relaxation, preferably in the less dense parts of the disc. In our particular setup, we have found most of the oscillating orbits to appear in the outer part of the disc and in the innermost one, below the initial inner radius. The KozaiLidov resonance is substantially affected by the presence of a global spherical potential (e.g. of an embedding cluster). In addition to its well-known damping influence on the KozaiLidov oscillations, however, we found that the presence of a moderately strong spherical potential may actually trigger these oscillations in the outer parts of the disc due to affecting the internal flow of angular momentum in the disc. The type of the Kozai-Lidov oscillations strongly depends on the particular setup. The same holds for the number of oscillating orbits which varies between the order of $\sim 10 \%$ in the most favorable case of an isolated disc and $\lesssim 1 \%$ for a disc embedded in a spherical potential (see Figure 6).

Direct consequence of the numerous oscillating orbits is modification of initial distributions of their orbital elements. From the observational point of view, the most interesting is the accelerated growth of root-mean-square inclinations with respect to the two-body relaxation driven evolution. We have found that in the case of an isolated disc, this process is the most prominent, being caused by the coplanar flipping. However, in this case, the highly inclined orbits lie geometrically in the thin disc structure. On the other hand, well above the original plane of the disc (up to $\pi / 2$ ) are pushed the oscillating orbits found in the models with intermediate mass of the embedding spherical cluster. Another possibly observable feature is the presence of highly eccentric orbits in the maxima of their Kozai-Lidov cycles.

The Kozai-Lidov oscillations themselves are difficult to be directly identified in observed astrophysical systems - resolution of our telescopes is not sufficient to determine the orbital elements of stars in foreign galactic nuclei. Even in the case of the Galactic Center, where the orbital elements of several stars have already been determined, the time-scale of the Kozai-Lidov oscillations exceeds the human life-time by several orders of magnitude. One possible indirect evidence of the Kozai-Lidov oscillations of stellar orbits in galactic nuclei (even the distant ones) are tidal disruptions of stars which are supposed to occur whenever the eccentricity and, consequently, the distance to the SMBH during the pericenter passage reaches a critical value. Being scaled to the nuclear stellar system of the Milky Way, the tidal disruption radius of a Solar-type star is of the order of $10^{-6} \mathrm{pc} \approx 2.5 \times 10^{-4} r_{\mathrm{u}}$, i.e. in the most favorable case of a disc not embedded in a spherical cluster, we predict somewhat more than one per cent of stars from the disc to undergo the tidal disruption event (see models M3 and M6 in Figure 6). Even though this is a relatively large number, it is also likely to expect numerous tidal disruptions of stars from the embedding spherical cluster due to the Kozai-Lidov cycles (Karas \& Subr 2007). Origin of a particular tidally disrupted star would thus be uncertain. Moreover, in contrary to Karas \& Subr (2007), in our current analysis, we have omitted general relativistic effects, such as the pericenter shift which is known to suppress the Kozai-Lidov oscillations (Blaes et al. 2002). In the context of the stellar disc around the SMBH, orbits reaching the stellar tidal disruption radii would suffer from this damping.

Within the context of our Galaxy, even more exciting appears to be tidal break-ups of binary stars which would occur at radii $\lesssim 10^{-3} \mathrm{pc}$, depending on their intrinsic orbital parameters. Tidal break-ups of binaries have already been suggested by Hills (1988) as a process that may bring stars to orbits tightly bound to the SMBH and, at the same time, accelerate their former binary companions to velocities greatly exceeding escape velocity from the Galaxy. Later on, both groups of stars were indeed found-the so-called S-stars (e.g. 
Ghez et al. 2005) which are young stars orbiting the central SMBH with the semi-major axes $\lesssim 0.04 \mathrm{pc}$ and the hyper-velocity stars which are young stars escaping from the Milky Way with velocities exceeding $500 \mathrm{kms}^{-1}$ (e.g. Brown et al. 2014). Here we suggest, and in a subsequent paper (Subr \& Haas in preparation further investigate, the possibility that all of them may have been born in a thin eccentric stellar disc and pushed to the tidal radius via the Kozai-Lidov oscillations.

\section{ACKNOWLEDGMENTS}

We thank David Vokrouhlický and the anonymous referee for helpful comments on the manuscript. We also acknowledge support of the Czech Science Foundation through the Project of Excellence No 14-37086G.

\section{REFERENCES}

Aarseth, S. J. 2003, Gravitational N-Body Simulations (Cambridge: Cambridge Univ. Press)

Alexander, R. D., Begelman, M. C., \& Armitage, P. J. 2007, ApJ, 654, 907

Bailey, M. E., Chambers, J. E., \& Hahn, G. 1992, A\&A, 257, 315

Bender, R., Kormendy, J., Bower, G., Green, R. et al. 2005, ApJ, 631, 280

Bertotti, B., Farinella, P., \& Vokrouhlický, D. 2003, Physics of the Solar System (Dordrecht: Kluwer)

Blaes, O., Lee, M. H., \& Socrates, A. 2002, ApJ, 578, 775

Bonnell, I. A., \& Rice, W. K. M. 2008, Sci, 321, 1060

Brown, W. R., Geller, M. J., \& Kenyon, S. J. 2014, ApJ, 787, 89

Cuadra, J., Armitage, P. J., \& Alexander, R. D. 2008, MNRAS, 388, L64

Gallardo, T., Hugo, G., \& Pais, P. 2012, Icar, 220, 392

Ghez, A. M., Salim, S., Hornstein, S. D. et al. 2005, ApJ, 620, 744

Gualandris, A., Mapelli, M., \& Perets, H. B. 2012, MNRAS, 427, 1793

Haas, J., Šubr, L., \& Vokrouhlický, D. 2011, MNRAS, 416, 1023

Hills, J. G. 1988, Natur, 331, 687

Hobbs, A., \& Nayakshin, S. 2009, MNRAS, 394, 191

Ivanov, P. B., Polnarev, A. G., \& Saha, P. 2005, MNRAS, 358, 1361

Karas, V., \& Šubr, L. 2007, A\&A, 470, 11

Katz, B., Dong, S., \& Malhotra, R. 2011, PhRvL, 107, 181101

Kocsis, B., \& Tremaine, S. 2011, MNRAS, 412, 187

Kozai, Y. 1962, AJ, 67, 591

Levin, Y., \& Beloborodov, A. M. 2003, ApJ, 590, L33

Li, G., Naoz, S., Kocsis, B., \& Loeb, A. 2014a, ApJ, 785, 116

Li, G., Naoz, S., Holman, M., \& Loeb, A. 2014b, ApJ, 791, 86

Lidov, M. L. 1962, Planet. Space Sci., 9, 719
Lithwick, Y., \& Naoz, S. 2011, ApJ, 742, 94

Löckmann, U., Baumgardt, H., \& Kroupa, P. 2009, MNRAS, 398, 429

Madigan, A.-M., Levin, Y., \& Hopman, C. 2009, ApJ, 697, L44

Mapelli, M., Hayfield, T., Mayer, L., \& Wadsley, J. 2012, ApJ, 749, 168

Morbidelli, A. 2002, Modern Celestial Mechanics (London: Taylor \& Francis)

Naoz, S., Farr, W. M., Lithwick, Y., Rasio, F. A., \& Teyssandier, J. 2013, MNRAS, 431, 2155

Nayakshin, S., Dehnen, W., Cuadra, J., \& Genzel, R. 2006, MNRAS, 366, 1410

Peiris, H. V., \& Tremaine, S. 2003, ApJ, 599, 237

Stewart, G. R., \& Ida, S. 2000, Icar, 143, 28

Šubr, L., Karas, V., \& Haas, J. 2007, IAUS, 238, 201

Šubr, L., \& Karas, V. 2005, in Hledík S., Stuchlík Z., eds, Proceedings of RAGtime 6/7: Workshops on Black Holes and Neutron Stars. Silesian University in Opava, Czech Republic, p. 281

Šubr, L., Schovancová, J., \& Kroupa, P. 2009, A\&A, 496, 695

Šubr, L., \& Haas, J. 2014, ApJ, 786, 121

Šubr, L., \& Haas, J. in preparation

Thomas, F., \& Morbidelli, A. 1996, CeMDA, 64, 209

Tremaine, S. 1995, AJ, 110, 628

Tremaine, S. 1998, AJ, 116, 2015

Vokrouhlický, D., \& Karas, V. 1998, MNRAS, 298, 53

Wardle, M., \& Yusef-Zadeh, F. 2008, ApJ, 683, L37 